\title{
HEMOGLOBINA GLICOSILADA Y SU RELACIÓN CON LA FRACCIÓN DE EYECCIÓN DEL VENTRICULO IZQUIERDO EN PACIENTES DIABÉTICOS TIPO 2 Y UN PRIMER INFARTO AGUDO DE MIOCARDIO
}

\author{
Maritza Pérez Mayorgai, Diego Gómez-Arbelaez', Enrique Melgarejoº, María Alejandra Bravo \\ Martínez', Luis Artemo González', Patricio López -Jaramillo? \\ ${ }^{1} M D$ Especialista en Medicina Interna y Endocrinología. Líder Grupo de Epidemiología Molecular \\ de Enfermedades Endocrinas. Profesor Asistente Universidad Militar Nueva Granada. \\ ${ }^{2} M D$ Dirección de Investigaciones, Fundación Oftalmológica de Santander - FOSCAL, Floridablanca, Colombia. \\ Instituto de Investigaciones MASIRA, Universidad de Santander - UDES \\ ${ }^{3}$ MD Especialista en Medicina Interna y Cardiología. Profesor Asociado Universidad Militar Nueva Granada \\ ${ }^{4}$ MD Residente de Medicina Interna. Universidad Militar Nueva Granada \\ ${ }^{5}$ MD Universidad Militar Nueva Granada. Residente Medicina Interna Universidad del Rosario \\ ${ }^{6}$ Ps Universidad Militar Nueva Granada. \\ ${ }^{7} \mathrm{MD}$ PhD FACP Especialista en Medicina Interna y Endocrinología. Dirección de Investigaciones, \\ Fundación Oftalmológica de Santander - FOSCAL, Floridablanca, Colombia. Instituto de Investigaciones \\ MASIRA, Universidad de Santander - UDES, Bucaramanga, Colombia.
}

Correspondencia: maritza.perez@unimilitar.edu.co

Recibido: Mayo 5 de 2014 Aceptado: Agosto 28 de 2014

\begin{abstract}
Resumen
Introducción: Dentro del espectro de las enfermedades cardiovasculares, la enfermedad isquémica del corazón se encuentra dentro de las dos patologías con mayor tasa de discapacidad y mortalidad. La Diabetes Mellitus tipo 2 es uno de los factores asociados a peor pronóstico en sujetos con esta enfermedad. Hoy en día se conoce que sobre el miocardio en isquemia, la hiperglicemia aumenta el estrés oxidativo, la hipoxia y por ende su necrosis.

Objetivo: Establecer si existe una relación entre los niveles de hemoglobina glicosilada de pacientes diabéticos tipo 2 con un primer infarto agudo de miocardio y la función del ventrículo izquierdo intrahospitalaria, medida por la fracción de eyección.

Materiales y Métodos: Estudio observacional en el que se realizó la caracterización de 36 pacientes con diabetes mellitus tipo 2 y un primer episodio de IAM hospitalizados en el Hospital Militar Central.

Resultados: Con el test de exactitud de Fischer se obtuvo que los pacientes diabéticos tipo 2 con primer infarto agudo de miocardio y valores de hemoglobina glicosilada por encima de 8,5\% presentaron una tendencia a tener fracciones de eyección por debajo de $50 \%(P=0,019)$.

Conclusiones: Se necesitan más estudios en los que se evalúen poblaciones más grandes de pacientes para lograr representar todo el espectro de valores de hemoglobina glicosilada y con ello, establecer un punto de corte más exacto a partir del cual sea válido sospechar una disminución de la función ventricular izquierda en el escenario de diabetes mellitus tipo 2 y un primer infarto agudo de miocardio.
\end{abstract}

Palabras clave: Infarto agudo de miocardio, hemoglobina glicosilada, fracción de eyección 


\title{
RELATIONSHIP BETWEEN GLYCATED HAEMOGLOBIN AND LEFT VENTRICULE EJECTION FRACTION IN DIABETES TYPE 2 PATIENTS ON THEIR FIRST ACUTE MYOCARDIAL INFARCTION
}

\begin{abstract}
Introduction: In the wide range of cardiovascular disease, ischemic heart disease is one of the mayor two pathologies that contribute with the greater disability and mortality rates. Type 2 Diabetes Mellitus is known to be one of the mayor risk factors associated with a worst prognosis among subjects who suffer from ischemic heart disease. Hyperglycemia augments oxidative stress in the ischemic myocardium and thus the hypoxemia and extent of necrosis.

Objective: To establish if there is a relationship between glycosylated hemoglobin levels and admissions left ventricular function, measured by ejection fraction, in type 2 diabetic patients suffering from a first acute myocardial infarction.

Materials and Methods: Observational study in which we characterized 36 type 2 diabetic patients suffering from a first acute myocardial infarction, hospitalized in The Hospital Militar Central.

Results: Using Fischer's exact test we found that that type 2 diabetics suffering from a first acute myocardial infarction and with glycosylated hemoglobin levels above $8.5 \%$ had a tendency to present an ejection fraction below 50\% $(\mathrm{P}=0.019)$.

Conclusions: Larger studies are needed in order to include all the possible values of glycosylated hemoglobin, so a more accurate cut off point from which a valid suspicion of deteriorated left ventricular function can be made, in the setting of type 2 diabetics with a first acute myocardial infarction.

Keywords: Acute myocardial infarction, glycosylated hemoglobin, ejection fraction.
\end{abstract}

\section{RELAÇÃO DA HEMOGLOBINA GLICADA COM A FRAÇÃO DE EJEÇÃO DO VENTRÍCULO ESQUERDO EM PACIENTES DIABÉTICOS TIPO 2 E UM PRIMEIRO INFARTO AGUDO DO MIOCÁRDIO.}

\begin{abstract}
Resumo
Introdução: Das doenças cardiovasculares, a doença isquêmica do coração e uma das duas patologias com maior taxa de discapacidade e mortalidade. A Diabetes Mellitus tipo 2 é um dos fatores associados ao pior prognóstico em pessoas com esta doença. Atualmente é conhecido que a hiperglicemia aumenta o estresse oxidativo, a hipóxia e a necroses no miocárdio isquêmico.

Objetivo: Estabelecer se existe uma relação entre os níveis de hemoglobina glicada de pacientes diabéticos tipo 2 com um primeiro infarto agudo do miocárdio (IAM) e a função do ventrículo esquerdo, segundo a fração de ejeção.

Materiais e Métodos: Estudo observacional, no qual foi feita a caracterização de 36 pacientes com Diabetes Mellitus tipo 2 e um primeiro IAM, hospitalizados no Hospital Militar Central. Resultados: Com o teste de Fischer, obteve-se uma tendência a frações de ejeção abaixo do $50 \%$ nos pacientes diabéticos tipo 2 com um primeiro IAM, e níveis de hemoglobina glicada acima de 8,5\% ( $P=0,019$.
\end{abstract}


Conclusões: Precisam-se mais estudos que avaliem um maior número de pacientes, com o fim de representar tudo o espetro de valores da hemoglobina glicada, estabelecendo um ponto de corte mais exato a partir do qual se possa suspeitar uma diminuição da função ventricular esquerda nos pacientes com diabetes mellitus tipo 2 e um primeiro IAM.

Palavras-chave: infarto agudo do miocárdio, hemoglobina glicada, fração de ejeção.

\section{Introducción}

Las enfermedades cardiovasculares (ECV) se han convertido en la principal causa de mortalidad en América Latina, incluso por encima de las enfermedades infecciosas $(1,2)$. En Colombia, el $28 \%$ de las muertes son causadas por ECV y representan la segunda causa de pérdida de años de vida saludable (3). Dentro del espectro de las ECV la enfermedad isquémica del corazón se encuentra dentro de las dos patologías con mayores tasas de discapacidad y mortalidad (1).

Diversos estudios han demostrado que la Diabetes Mellitus tipo 2 (DM2) es uno de los principales factores asociados a peor pronóstico en sujetos con enfermedad ateroesclerótica $(4,5)$. Múltiples estudios prospectivos han encontrado que los individuos diabéticos tienen dos a cuatro veces mayor mortalidad por la enfermedad arterial coronaria (CAD) en diferentes grupos étnicos y raciales (6,7). La diabetes también aumenta la probabilidad de tener enfermedad aterosclerótica carotídea severa (8-11) y la mortalidad por accidente cerebrovascular se incrementa casi tres veces en los pacientes diabéticos $(11,12)$.

La asociación entre hiperglicemia y CAD ha sido por muchos años atribuida a la predisposición que normalmente presentan los individuos con resistencia a la insulina o hiperglicemia de desarrollar DM2. Sin embargo, la hiperglicemia per se, independientemente de otros factores de riesgo cardiovascular puede estar implicada en el desarrollo de ateroesclerosis por sus efectos nocivos sobre la estructura y el metabolismo endotelial (13-30).

En el escenario de un infarto agudo de miocardio (IAM), el papel de la hiperglicemia no está aún del todo claro, para muchos es un marcador del estrés que sufre el organismo durante el evento y por ende, el resultado de la descarga simpática de catecolaminas y adrenal de cortisol. Sin embargo, se conoce del efecto deletéreo de la hiperglicemia sobre el miocardio en isquemia; en presencia de ésta, aumenta el estrés oxidativo, la hipoxia y por ende, la necrosis del miocardio (31).

En los estados de anaerobiosis a los que se enfrenta el miocardio en isquemia, su metabolismo cambia la fuente de energía principal de ácidos grasos libres a glucosa. Este cambio se ve relacionado con el aumento de transportadores GLUT4 en los miocitos para conseguir la entrada de mayor cantidad de glucosa y así un aumento en la glicolisis (32). Sin embargo, este mecanismo de reducción del consumo de oxígeno en el miocardio hipóxico o isquémico se ve truncado en presencia de resistencia a la insulina, que disminuye la disponibilidad de glucosa dentro de la célula miocárdica, obligándola a incrementar su demanda de oxígeno para conseguir la oxidación de ácidos grasos libres como fuente de energía. El resultado es mayor acidosis intracelular, mayor necrosis e hiperglicemia $(33,34)$.

Lo anterior explica en parte por qué los pacientes con DM2, cuya patología se basa en la resistencia a la insulina tienen mayor riesgo de severidad (35-37) y peor pronóstico $(38,39)$ de IAM, Pues, dentro de este grupo, el riesgo es aún mayor. El efecto nocivo de la hiperglicemia crónica, tomado de los valores de hemoglobina glicosilada, se ha postulado por algunos como un factor de riesgo independiente de CAD, especialmente para mujeres. $(40,41)$.

La fracción de eyección como medida de la función ventricular izquierda, se ha empleado como factor pronóstico a largo plazo en síndrome coronario agudo (31). Sin embargo, su medición intrahospitalaria ha sido usada como método para estimar el tamaño y por ende la severidad del evento coronario. A mayor severidad, mayor tiempo de estancia hospitalaria y mayor morbimortalidad en la misma $(33,34)$.

Este estudio pretende establecer si existe una relación entre los niveles de hemoglobina glicosilada de pacientes diabéticos tipo 2 con un primer infarto agudo de miocardio y la fracción de eyección inicial (en las primeras 72 horas) del ventrículo izquierdo tras evento coronario.

\section{Materiales y Métodos}

Estudio observacional, en el que se realizó la caracterización de pacientes con diabetes mellitus tipo 2 y un primer episodio de IAM hospitalizados en el Hospital Militar 
Central. Entre Septiembre de 2009 y Octubre de 2012 se reclutaron 36 pacientes.

Se definió IAM si estaban presentes todos los siguientes parámetros: elevación de troponina sérica ultrasensible (con un valor por encima del percentil 99 del límite de referencia superior), síntomas clínicos de isquemia (dolor y/o sensación de opresión precordial, irradiación del dolor al miembro superior izquierdo, diaforesis y síntomas disautonómicos), y al menos uno de tres signos clínicos de isquemia: cambios en electrocardiograma (ECG) (nuevos cambios ST-T ó nuevo bloqueo de rama izquierda), desarrollo de ondas Q patológicas en el ECG o evidencia en imágenes de nueva pérdida de miocardio viable o nueva alteración de movimiento de pared regional en el electrocardiograma.

Cada paciente expresó su deseo de participar en el estudio con firma de consentimiento informado. La realización del estudio fue aprobada por el comité de ética del Hospital Militar Central. Durante su estancia hospitalaria a cada paciente se le realizó una entrevista verbal donde se consignaron datos de edad, sexo, raza, procedencia urbana o rural, nivel educativo, tiempo transcurrido entre el inicio de los síntomas y asistencia a lugar de primera atención hospitalaria, antecedentes personales y familiares de HTA, angina, DM, $\mathrm{ECV}$, enfermedad arterial periférica, antecedentes familiares de IAM y si se encontraban en tratamiento con antidiabéticos orales $\mathrm{y} / \mathrm{o}$ insulina. Se tomaron medidas físicas de talla, peso y perímetro abdominal y de cadera. Se revisaron las historias clínicas de los pacientes para obtener los valores de troponina sérica ultrasensible, tipo de infarto y localización, niveles de hemoglobina glicosilada (dentro del mes anterior al evento) y la fracción de eyección del ventrículo izquierdo por ecocardiograma transtorácico (MM 2D DOPPLER COLOR) realizado dentro de las primeras 72 horas de ingreso.

El análisis estadístico se hizo por medio del programa SPSS, la parte descriptiva se hizo por medio de distribuciones de frecuencia para variables nominales y ordinales; las variables numéricas se describieron por medio de medidas de tendencia central y dispersión. Igualmente se hicieron correlaciones de tipo paramétrico (Pearson) y no parámétricas (Fischer).

\section{Resultados}

En total se reclutaron 106 pacientes con primer IAM, de estos 36 pacientes tenían diagnóstico previo de DM2 y cumplieron los criterios de inclusión del estudio.
Las características de la población se encuentran en la tabla 1 . De los 36 pacientes 11 eran mujeres, todas con menopausia establecida. La edad promedio de los pacientes fue de 67,6 años; el 47,2\% de los pacientes presentó un IAM con elevación del segmento ST y el $52,8 \%$ tuvo un IAM sin elevación del segmento ST. De éstos, el 88\%y el $90 \%$ respectivamente, tuvieron una severidad calificada en killip I, el resto de pacientes tuvo una severidad calificada en killip 2; en esta cohorte no hubo pacientes con infarto killip III ni IV. El 91,7\% de los pacientes era de raza mestiza y provenían de un área urbana en un $88,9 \%$.

Tabla 1. Características De La Población

\begin{tabular}{|l|r|c|}
\hline \multicolumn{1}{|c|}{ Característica } & Frecuencia & Porcentaje \\
\hline Sexo & 25 & $69.4 \%$ \\
Hombre & 11 & $30.6 \%$ \\
Mujer & 32 & \\
\hline Procedencia & 4 & $88.9 \%$ \\
Urbano & $11.1 \%$ \\
Rural & 2 & \\
\hline Raza & 1 & $5.6 \%$ \\
Blanco & 33 & $2.8 \%$ \\
Negro & 1 & $91.7 \%$ \\
Mestizo & 10 & $2.8 \%$ \\
\hline Nivel Educativo & 11 & $27.8 \%$ \\
Ninguno & 8 & $30.6 \%$ \\
1-5 años & 6 & $22.2 \%$ \\
6-11 años & 17 & $16.7 \%$ \\
Técnico & 19 & $47.2 \%$ \\
Universitario & 27 & $52.8 \%$ \\
\hline IAMEST & 4 & $93.1 \%$ \\
\hline IAMSEST & 1 & $13.8 \%$ \\
\hline Ant. HTA & 16 & $3.4 \%$ \\
\hline Ant. Angina & & $55.2 \%$ \\
\hline Ant. ECV & & \\
\hline Ant. Tabaquismo & & \\
\hline
\end{tabular}

IAMEST: Infarto Agudo Del Miocardio Con Elevación Del ST, IAMSEST: Infarto Agudo Del Miocardio Sin Elevación Del ST, HTA: Hipertensión Arterial, ECV: Enfermedad Cerebro Vascular.

En la tabla 2 se encuentran los valores de las variables estudiadas en los 36 pacientes. El 28\% ( $n=10)$ de los pacientes, tenían tratamiento hipolipemiante previo a su ingreso; de estos, el 90\% tenían niveles de colesterol total y colesterol LDL dentro de metas $(<200$ y $100 \mathrm{mg} / \mathrm{dl}$ respectivamente), el $60 \%$ los niveles de triglicéridos dentro de metas $(<150 \mathrm{mg} / \mathrm{d}$ ); solo un paciente, tenía el nivel de colesterol HDL en el rango recomendado. El 25\% de los pacientes se encontraban en metas para perímetro abdominal, para el que se tomó como punto de corte $90 \mathrm{~cm}$ 
Tabla 2. Variables Medidas

\begin{tabular}{|l|l|r|r|r|r|}
\hline \multicolumn{1}{|c|}{ Variables } & N & Mínimo & Máximo & Media & \multicolumn{1}{c|}{ SD } \\
\hline Edad (años) & 36 & 43 & 86 & 67.69 & 9.12 \\
\hline Años con DM & 36 & 1 & 26 & 8.81 & 6.598 \\
\hline GB (mg/dl) & 36 & 52 & 346 & 145.61 & 61.74 \\
\hline Hb1AC (\%) & 36 & 5.48 & 10.32 & 7.48 & 1.15 \\
\hline FE (\%) & 36 & 25 & 67 & 52.11 & 9.27 \\
\hline CT (mg/dl) & 36 & 91 & 302 & 153.86 & 45.02 \\
\hline LDL (mg/dl) & 36 & 39.0 & 162.0 & 89.31 & 32.14 \\
\hline HDL (mg/dl) & 36 & 24 & 60 & 37.66 & 8.21 \\
\hline VLDL (mg/dl) & 36 & 15.4 & 103.4 & 30.23 & 16.61 \\
\hline TG (mg/dl) & 36 & 77 & 517 & 151.92 & 82.63 \\
\hline IMC (kg/m2) & 36 & 17.1 & 38.0 & 25.64 & 4.11 \\
\hline PABD (cm) & 36 & 65.86 & 109.25 & 94.43 & 10.22 \\
\hline
\end{tabular}

GB: Glicemia Basal, Hb1AC: Hemoglobina Glicosilada, FE: Fracción De Eyección, CT: Colesterol Total, LDL: Colesterol De Baja Densidad, HDL: Colesterol De Alta Densidad, VLDL: Colesterol de Muy Baja Densidad TG: Triglicéridos, IMC: Índice De Masa Corporal, PABD: Perímetro Abdominal.

para hombres y $80 \mathrm{~cm}$ para mujeres. En la tabla $3 \mathrm{se}$ encuentran los porcentajes de cumplimiento de metas en términos de perfil lipídico y perímetro abdominal para la totalidad de los pacientes.

Tabla 3. Metas Metabólicas

\begin{tabular}{|l|c|c|}
\hline \multicolumn{1}{|c|}{ Meta } & Frecuencia & Porcentaje \\
\hline CT $(<200 \mathrm{mg} / \mathrm{dl})$ & 31 & $86 \%$ \\
\hline LDL $(<100 \mathrm{mg} / \mathrm{dl})$ & 23 & $64 \%$ \\
\hline TG $(<150 \mathrm{mg} / \mathrm{dl})$ & 24 & $67 \%$ \\
\hline PABD $(<80 \mathrm{~cm} \mathrm{M},<90 \mathrm{~cm} \mathrm{H})$ & 9 & $25 \%$ \\
\hline
\end{tabular}

M: Mujeres, H: Hombres.

El test de correlación de Pearson no evidenció correlación estadísticamente significativa $(r=-0,15$ y $\mathrm{p}=0,35)$ entre los niveles hemoglobina glicosilada y FE; sin embargo, al separar en dos grupos a los pacientes, de acuerdo a sus niveles de hemoglobina glicosilada (en menores y mayores de $8.5 \%$, punto de corte elegido por haber sido él que mejor discriminó la relación de hemoglobina glicolisada con FE) y FE ( en menores y mayores de 50\%) y realizar el test de exactitud de Fischer, se determinó que, con valores de hemoglobina glicosilada por encima de 8,5\% había una tendencia estadísticamente significativa de presentar fracciones de eyección por debajo de $50 \%(P=0,019)$, como se observa en la gráfica 1 .

Fue realizada un test de correlación Mantel-Haenszel para determinar si la presencia de hipertensión, el número de

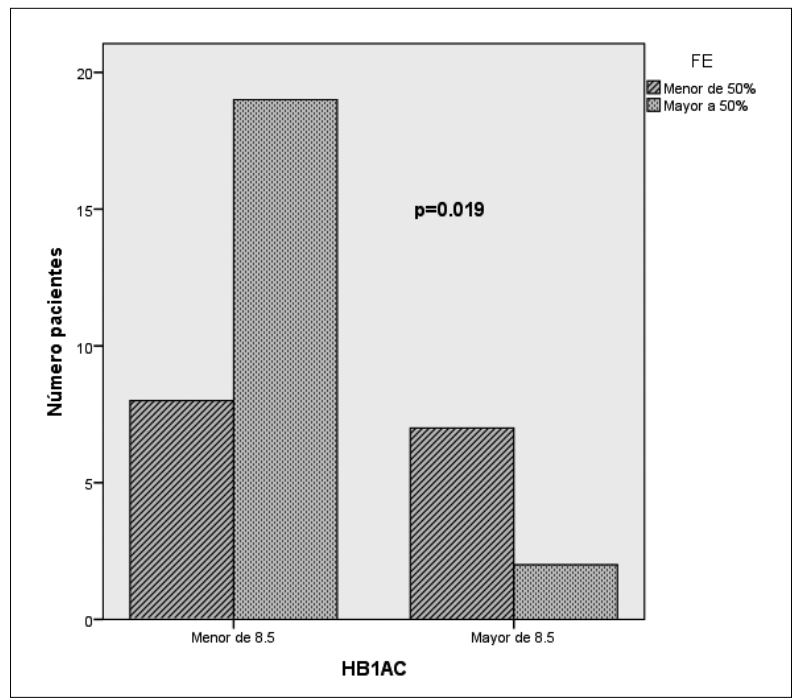

Grafica 1. Relación entre Hemoglobina Glicosilada y Fracción de Eyección.

años desde el diagnóstico de diabetes, el pertenecer al sexo femenino o el uso previo de inhibidores de enzima convertidora de angiotensina podían modificar la relación entre el nivel de Hemoglobina Glicosilada y la fracción de eyección. Al ser un ajuste a estas variables no se modificó la relación entre la hemoglobina glicosilada y la fracción de eyección. Tabla 4.

Tabla 4. Resultados Test de Mantel -Haenszel

\begin{tabular}{|l|c|c|}
\hline \multicolumn{1}{|c|}{ Variable } & $\mathbf{X}^{\mathbf{2}}$ & Valor $\mathbf{p}$ \\
\hline Hipertensión arterial & 3.481 & 0,042 \\
\hline Años con Diabetes Mellitus tipo 2 & 4.291 & 0,038 \\
\hline Sexo & 4.443 & 0,035 \\
\hline Uso de IECAS & 4.078 & 0,043 \\
\hline
\end{tabular}

\section{Discusión}

El pobre control de la DM2 y su consecuente estado de hiperglicemia, no solo predisponen a un aumento en el riesgo de presentar eventos coronarios sino además un incremento en la severidad de dicho evento (51).

Como se mencionó anteriormente el papel exacto de la hiperglicemia en el escenario de un IAM no es aún del todo claro. Aunque muchos consideran que su presencia es el reflejo del estado de estrés y la consecuente liberación de hormonas contrarreguladoras (31), la evidencia bioquímica de los cambios en el metabolismo del miocar- 
dio isquémico, sugieren que también puede ser causante la severidad del evento y que dicho efecto es aún más deletéreo en presencia de resistencia a la insulina $(33,34)$.

Siguiendo la lógica de la anterior premisa, este estudio quiso demostrar si existe una relación o algún valor a partir del cual los niveles de hemoglobina glicosilada de un paciente diabético y un primer IAM podrían predecir una pobre función ventricular izquierda, medida por la FE con ecocardiograma transtorácico dentro de las primeras 72 horas posteriores al ingreso y con ello mayor riesgo de morbimortalidad intrahospitalaria.

Aunque con el test de correlación de Pearson se estableció que existe una relación débil y estadísticamente no significativa entre hemoglobina glicosilada y FE, el test de exactitud de Fisher evidenció que con niveles de hemoglobina glicosilada iguales o mayores a 8.5\% la FE tiende a estar por debajo del $50 \%$ en los pacientes diabéticos con primer IAM y que dicha tendencia es estadísticamente significativa y desaparece con valores de hemoglobina glicosilada menores o iguales a $8.1 \%$.

Lo anterior deja una brecha de incertidumbre para aquellos pacientes con niveles de hemoglobina glicosilada entre 8.2 y $8.4 \%$. Una limitación para nuestro estudio fue que dentro de los 36 pacientes que cumplieron criterios de inclusión, ninguno tuvo valores de hemoglobina glicosilada dentro de este rango. Se necesitan estudios más grandes en los que se logren representar todos los posibles valores de hemoglobina glicosilada y por ende obtener un punto de corte más exacto.

Otra de las limitaciones del estudio es el no haber ajustado el hallazgo de la tendencia de FE menor de 50\% con hemoglobinas Glicosiladas mayores a $8,5 \%$ por las otras variables que pueden contribuir a una FE deteriorada en el contexto de pacientes diabéticos con primer IAM.

Teniendo en cuenta que, a mayor tiempo con DM2 habrá mayor disfunción endotelial y por ende, en presencia de un IAM,cabría esperar un peor desenlace en la función ventricular izquierda $(20,24,51)$, se realizó el test de correlación de Pearson y el test de exactitud de Fischer para establecer si existe una tendencia para presentar peor FE en el primer IAM entre más años con diagnóstico de DM2; sin embargo, nuestros resultados fueron negativos en las dos pruebas.

Creemos que estos resultados pudieron deberse en parte a la falta de información que tienen los pacientes acerca de su enfermedad y el tiempo que llevan con la misma. Se debe tener en cuenta que el $61.2 \%$ de los participantes en este estudio tenían máximo 11 años de escolaridad y su bajo conocimiento de la enfermedad pudo restar valor a la relación entre las variables estudiadas.

En cuanto a los parámetros de otras metas metabólicas que se midieron en los pacientes, se evidenció que, al menos una tercera parte no se encontraban controlados al momento de su evento coronario, contribuyendo seguramente a la presentación y severidad del mismo.

El perímetro abdominal como medida indirecta de la grasa visceral y con ello de resistencia a la insulina, fue el parámetro con menos pacientes dentro de metas y sólo el $28 \%$ de los pacientes estaba en manejo con hipolipemiantes previo a su ingreso. Lo anterior demuestra que la prevención primaria, tanto farmacológica como en estilos de vida, está siendo ineficiente y continúa contribuyendo a la morbimortalidad cardiovascular de la DM2.

\section{Conclusiones}

Existe una tendencia estadísticamente significativa, en pacientes con DM2 y primer IAM de presentar una FE inicial deteriorada cuando tienen niveles de hemoglobina glicosilada por encima de $8.5 \%$. Se necesitan más estudios en los que se evalúen poblaciones más grandes de pacientes para lograr representar todo el espectro de valores de hemoglobina glicosilada y con ello, establecer un punto de corte más exacto a partir del cual sea válido sospechar una disminución en la función inicial del ventrículo izquierdo y por ende mayor morbimortalidad intrahospitalaria en el escenario de DM2 con un primer IAM.

Conflicto de intereses: Ninguno reportado por los autores

Financiación: Universidad Militar Nueva Granada, Fundación Oftalmológica de Santander FOSCAL.

\section{Referencias}

1. Organización Panamericana de la Salud y Organización Mundial de la Salud. Enfermedades Cardiovasculares, especialmente la hipertensión arterial. 2000. Whashington D.C.

2. Murray CJ, Lopez AD. Mortality by cause for eight regions of the world: Global Burden of Disease Study. Lancet 1997;349:1269-76.

3. Minisalud. La carga de la enfermedad en Colombia. 1994. Colombia.

4. Rosamond W, Flegal K, Friday G, Furie K, Go A, Greenlund K. Heart disease and stroke statistics 2007 update: a report from the American Heart Association Statistics Committee and Stroke Statistics Subcommitee. Circulation 2007;115:e69-171. 
5. Luscher TF, Creager MA, BEckman JA, Consentino F. Diabetes vascular disease: pathophysiology, clinical consequences, and medical therapy: Part II. Circulation. 2003;108:1655-1661.

6. American Diabetes Association. Consensus statement: role of cardiovascular risk factors in prevention and treatment of macrovascular disease in diabetes. Diabetes Care 1993;16:72-78.

7. Pyorala K, Laakso M, Uusitupa M. Diabetes and atherosclerosis: an epidemiologic view. Diabetes Metab Rev 1987;3:463-524.

8. Cubillos-Garzón LA, Casas JP, Morillo CA, Bautista LE. Congestive heart failure in Latin America: the next epidemic. Am. Heart J.2004;147:412-17.

9. Lopez-Jaramillo P, Casas JP, Bautista LE, Serrano N, Morillo CA. An integrated proposal to explain the epidemic of cardiovascular disease in a developing country: From socio-economic factors to free radicals. Cardiology 2001;96:1-6.

10. O'Leary DH, Polak JF, Cronmal RA, et al. Distribution and correlates of sonographically detected carotid artery disease in the Cardiovascular Health Study. The CHS Collaborative Research Group. Stroke 1992;23:1752-1760.

11. Folsom AR, Eckfeldt JH, Weitzman S, et al. Relation of carotid artery wall thickness to diabetes mellitus, fasting glucose and insulin, body size, and physical activity. Atherosclerosis Risk in Communities (ARIC) Study Investigators. Stroke 1994;25:66-73.

12. Stamler J, Vaccaro O, Neaton JD, et al. Diabetes, other risk factors, and 12-yr cardiovascular mortality for men screened in the Multiple Risk Factor Intervention Trial. Diabetes Care 1993;16:434-444.

13. Hadi HA, Al Suwaidi J, Bener A, Khinji A, Al Binali HA. Thrombolytic therapy use for acute myocardial infarction and outcome in Qatar. Int J Cardiol 2005;102:249-54.

14. Luscher TF, Creager MA, Beckman JA, Cosentino F. Diabetes vascular disease: pathophysiology, clinical consequences, and medical therapy: Part II. Circulation. 2003; 108: 1655-1661.

15. Kjaergaard SC, Hansen HH, Fog L, Builow I, Christensen PD. In-hospital outcome for diabetic patients with acute myocardial infarction in the thrombolytic era. Scand Cardiovasc J. 1999; 33: 166-170

16. Haffner SM, Lehto S, Rönnemaa T, Pyörälä K, Laakso M. Mortality from coronary heart disease in subjects with type 2 diabetes and in nondiabetic subjects with and without prior myocardial infarction. N Engl J Med. 1998;339: 229-234.

17. Herlitz J, Karlson BW, Lindqvist J, Sjölin M. Rate and mode of death during five years of follow-up among patients with acute chest pain with and without a history of diabetes mellitus. Diabet Med. 1998; 15: 308-314.

18. Kosuge M, Kimura K, Kojima S, Sakamoto T, Matsui K, Ishihara $\mathrm{M}$, et al. Effects of glucose abnormalities on in-hospital outcome after coronary intervention for acute myocardial infarction. Circ J 2005; 69: 375-379.

19. Harjai KJ, Stone GW, Boura J, Mattos L, Chandra H, Cox D, et al. Comparison of outcomes of diabetic and nondiabetic patients undergoing primary angioplasty for acute myocardial infarction. Am J Cardiol 2003; 91: 1041- 1045.

20. Mukamal KJ, Nesto RW, Cohen MC, Muller JE, Maclure M, SherwoodJB, et al. Impact of diabetes on long-term survival after acute myocardial infarction: Comparability of risk with prior myocardial infarction. DiabetesCare 2001; 24: 1422- 1427.
21. Folsom AR, Rasmussen ML, Chambless LE, Howard G, Cooper LS, Schmidt MI, Heiss G. Prospective associations of fasting insulin, body fat distribution, and diabetes with risk of ischemic stroke. TheAtherosclerosis Risk in Communities (ARIC) Study Investigators. Diabetes Care. 1999; 22: 1077-1083.

22. Keavney BD, Dudley CR, Stratton IM, et al. UK prospective diabetes study (UKPDS) 14: association of angiotensin-converting enzyme insertion/deletion polymorphism with myocardial infarction in NIDDM. Diabetologia 1995;38: 948-952.

23. Kannel WB, McGee DL. Diabetes and cardiovascular disease. The Framingham Study. JAMA 1979;241:2035-2038.

24. Jarrett RJ, McCartney P, Keen $\mathrm{H}$. The Bedford survey: ten year mortality rates in newly diagnosed diabetics, borderline diabetics and normoglycaemic controls and risk indices for coronary heart disease in borderline diabetics. Diabetologia 1982;22:79-84.

25. Jarrett RJ, Shipley MJ. Type 2 (non-insulin-dependent) diabetes mellitus and cardiovascular disease putative association via common antecedents; further evidence from the Whitehall Study. Diabetologia 1988;31:737-740.

26. Fontbonne A, Eschwege E, Cambient F, et al. Hypertriglyceridaemia as a risk factor of coronary heart disease mortality in subjects with impaired glucose tolerance or diabetes. Results from the 11-year follow-up of the Paris Prospective Study. Diabetologia 1989;32:300-304.

27. Nathan DM. Long-term complications of diabetes mellitus. N Engl J Med 1993;328:1676-1685.

28. Barrett-Connor E, Wingard DL. Sex differential in ischemic heart disease mortality in diabetics: a prospective population-based study. Am J Epidemiol 1983;118:489-496.

29. Reaven G. Syndrome X: 10 years after. Drugs 1999;58[Suppl 1]:19-20.

30. Executive Summary of the Third Report of the National Cholesterol Education Program (NCEP). Expert Panel on Detection, Evaluation, and Treatment of High Blood Cholesterol in Adults (Adult Treatment Panel III). JAMA 2001;285:2486-2497.

31. de Mulder M, Umans VA, Stam F, Cornel JH, Oemrawsingh RM, Boersma E. Intensive management of hyperglycaemia in acute coronary syndromes. Study design and rationale of the BIOMArCS 2 glucose trial. Diabet Med. 2011 Oct;28(10):1168-75.

32. Sun D, Nguyen N, DeGrado TR, Schwaiger M, Brosius FC III. Ischemia induces translocation of the insulin-responsive glucose transporter GLUT4 to the plasma membrane of cardiac myocytes. Circulation 1994; 89: 793-798.

33. Dandona P, Chaudhuri A, Ghanim H, Mohanty P. Effect of hyperglycemia and insulin in acute coronary syndromes. Am J Cardiol 2007; 99: $12 \mathrm{H}-18 \mathrm{H}$.

34. Marfella R, Di Filippo C, Portoghese M, Ferraraccio F, Rizzo MR, Siniscalchi $\mathrm{M}$ et al. Tight glycemic control reduces heart inflammation and remodeling during acute myocardial infarction in hyperglycemic patients. J Am Coll Cardiol 2009; 53: 1425-1436.

35. Krolewski AS, Warram JH, Rand LI, et al. Epidemiologic approach to the etiology of type I diabetes mellitus and its complications. N Engl J Med 1987; 317:1390-1398.

36. Asagami T, Li W, Abbasi FA, Tsao PS et al. Metformin attenuates plasma asymmetric dimethylarginine and monocyte adhesion in type 2 diabetes. Circulation 1999;102[Suppl II]:1129(abst). 
37. Vigorita VJ, Moore GW, Hutchins GM. Absence of correlation between coronary arterial atherosclerosis and severity or duration of diabetes mellitus of adult onset. Am J Cardiol 1980;46:535-542.

38. Aronson D. Pharmacologic modulation of autonomic tone: implications for the diabetic patient. Diabetologia 1997;40:476-481.

39. Mukamal KJ, Nesto RW, Cohen MC, et al. Impact of diabetes on long-term survival after acute myocardial infarction: comparability of risk with prior myocardial infarction. Diabetes Care 2001;24:1422-1427.

40. Rodriguez BL, Lau N, Burchfiel CM, et al. Glucose intolerance and 23-year risk of coronary heart disease and total mortality: the Honolulu Heart Program. Diabetes Care 1999;22:1262-1265.

41. Singer DE, Nathan DM, Anderson KM, et al. Association of HbA1c with prevalent cardiovascular disease in the original cohort of the Framingham Heart Study. Diabetes 1992;41:202-208.

42. Andersen NH, Hansen TK, Christiansen JS. Changes in glycaemic control are related to the systolic function in type 1 diabetes mellitus. Scand Cardiovasc J. 2007 Apr;41(2):85-8. Clin Sci (Lond). $2006 \mathrm{Jul} ; 111(1): 53-9$.

43. Marfella R, Di Filippo C, Portoghese M, Ferraraccio F, Rizzo MR, Siniscalchi M, Musacchio E, D'Amico M, Rossi F, Paolisso G. Tight glycemic control reduces heart inflammation and remodeling during acute myocardial infarction in hyperglycemic patients. J Am Coll Cardiol. 2009 Apr 21;53(16):1425-36.

44. Capes SE, Hunt D, Malmberg K, Gerstein HC. Stress hyperglycaemia and increased risk of death after myocardial infarction in patients with and without diabetes: a systematic overview. Lancet 2000;355:773-8.
45. Malmberg K, Ryden L, Efendic S, et al. Randomized trial of insulin-glucose infusion followed by subcutaneous insulin treatment in diabetic patients with acute myocardial infarction (DIGAMI study): effects on mortality at 1 year. J Am Coll Cardiol 1995;26:57- 65.

46. Deedwania P, Kosiborod M, Barrett E, et al. American Heart Association Diabetes Committee of the Council on Nutrition, Physical Activity, and Metabolism. Hyperglycemia and acute coronary syndrome: a scientific statement from the American Heart Association Diabetes Committee of the Council on Nutrition, Physical Activity, and Metabolism. Circulation 2008;117:1610-9.

47. McGuire DK, Newby LK, Bhapkar MV, et al.; SYMPHONY and 2nd SYMPHONY Investigators. Association of diabetes mellitus and glycemic control strategies with clinical outcomes after acute coronary syndromes. Am Heart J 2004; 147: 246-52.

48. Rasoul S, Ottervanger JP, Timmer JR, et al. Zwolle myocardial infarction study group. Impact of diabetes on outcome in patients with non-ST-elevation myocardial infarction. Eur J Intern Med 2011; 22: 89-92.

49. Ergelen M, Uyarel H, Cicek G, et al. Which is worst in patients undergoing primary angioplasty for acute myocardial infarction? Hyperglycaemia? Diabetes mellitus? Or both? Acta Cardiol 2010; 65: 415-23

50. Cao JJ, Hudson M, Jankowski M, et al. Relation of chronic and acute glycemic control on mortality in acute myocardial infarction with diabetes mellitus. Am J Cardiol 2005; 96: 183-6.

51. Johnstone MT, Kinzfogl GP. Chapter 28: Diabetes Mellitus and Heart Disease. Contemporary Cardiology: Diabetes and Cardiovascular Disease, Second Edition. Human Press 2005. 